\title{
Caso clínico: mala adherencia al tratamiento de una gestante en hemodiálisis
}

\author{
María Jesús Ávarez López, María Teresa Torres Vázquez, Ana Botella Lorenzo, Alfonso de la Vara Almonacid
}

\section{Centro de hemodiálisis Los Lauros. Majadahonda. Madrid}

\section{Introducción:}

Uno de los acontecimientos más gratificantes que pueden ocurrir en una unidad de hemodiálisis es seguir la evolución de una gestación exitosa. Se trata de un proceso que requiere la actuación de un equipo multidisciplinar formado por gran variedad de especialidades sanitarias en total coordinación. Nuestro caso trata de describir las actuaciones de los profesionales, siempre desde una perspectiva enfermera, durante la gestación de una paciente en programa de hemodiálisis con gran problemática social, que incumplía reiteradamente nuestras recomendaciones casi siempre por desconfianza y ansiedad, y cómo, a pesar de todas las dificultades, resultó un parto vaginal sin complicaciones a las treinta y cuatro semanas de gestación. A lo largo del texto describimos el curso del embarazo, así como los problemas que se nos fueron presentando; tales como la infección de orina, infección respiratoria, descontrol del peso seco, aumento de edemas, hiperparatiroidismo, anemia, hipertensión arterial, coagulación de circuitos y malfuncionamiento de catéter. Dentro de los diagnósticos de enfermería detectamos los siguientes: conflicto de decisiones, ansiedad, afrontamiento inefectivo del régimen terapéutico, temor, riesgo de violencia dirigida a otros, riesgo de desequilibrio del volumen de líquidos, exceso de volumen de líquidos y riesgo de deterioro de la vinculación entre los padres y el lactante. Finalmente concluimos la importancia de la actuación conjunta de los equipos en la consecución de objetivos y el peso que adquieren los cuidados de enfermería en la vigilancia y control de los riesgos potenciales y reales en un caso sin precedente en nuestra unidad.

\section{Referencias Bibliográficas}

1. Embarazo en mujeres en diálisis crónica: revisión. Karina R. Furaz-Czerpak, Gema Fernández-Juárez, M. Ángeles Moreno-de la Higuera, Elena Corchete-Prats, Adriana Puente-García, Roberto MartínHernández. Rev. Nefrol. 28Feb.2012.Vol.32 n³.

2. Gestación con éxito en paciente con hipotiroidismo en programa de diálisis. Consuelo Carretero Sierra, Ana Isabel García González, Cristina Gómez Menor, Laura Hilara Rico, Mariana Lorite Godoy, Lourdes Marcos Ayala, María Medina Real, Olimpia Ortega Marcos. Rev. Soc. Esp. Enferm. Nefrol. V.9 n.4 Madrid oct-dic 2006.

3. Gestación con éxito en una paciente con IRC en programa de hemodiálisis. Luque Vadillo Emilia, Matamala Gaston Ana, Places Balsalobre Jaime, Alconchel Cabezas Silvia, Torres Jausà María, Díaz Cocera María. Rev. Soc. Esp. Nefrol.2002;19:47/49.

4. Diálisis y embarazo, 13 años de experiencia en el hospital público. Amelia R. Bernasconi, Alicia Mobel Lapidus, Rosa Waisman, Amador A. Liste, Liliana S. Vote, Ricardo M. Heguilén. Revista de nefrología, diálisis y trasplante. Vol 27-n³-2007.

5. Pregnancy in Dialysis Patients: Is the Evidence Strong Enough to Lead us to Change Our Counseling Policy? Giorgina Barbara Piccoli, Anne Conijn, Valentina Consiglio, Elena Vasario, Rosella Attini, María Chiara Deagostini, Salvatore Bontempo and Tullia Todros. Rev. Clin. J. Am. Soc. Nephrol 5: 6271, 2010. 
6. Autocuidados, abordaje holístico y trabajo en equipo elementos facilitadores para la consecución de un embarazo a-término en paciente en hemodiálisis. Carme Moreno Aliaga, Inmaculada Fonfría Perez, Rosa Moll Guillén, Laura Navarro Daudén, Anna Martí i Monros, Julia Farga Badal. Rev. Enferm. Nefrol 2012; suppl(1): 97/101.
7. Diálisis y embarazo. María José Marqués Racionero, Virginia Marín Fábregas, María Luisa Molina Conde. Rev. Enf. Nefrol. 2012; 15 Suppl(1): 102/141.

8. Cómo escribir un caso clínico en enfermería utilizando Taxonomía NANDA, NOC, NIC. Index Enferm. Vol.20 nºl-2 Granada ene.-jun.2011. 